\title{
Thermal imaging in automatic rodent's social behaviour analysis
}

by M. Mazur-Milecka*

\author{
* Gdansk Univ. of Technology, 80-233, Narutowicza Str., Gdansk, Poland, magmilec@pg.gda.pl
}

\begin{abstract}
Laboratory rodent social behaviour analysis is an extremely important task for biological, medical and pharmacological researches. In this work thermal images features that facilitate analysis are presented. Methods to distinguish objects on the basis of thermal distribution are tested. Actions of grooming or biting one rodent by another important social behaviour incidents - are clearly visible in thermal images due to the saliva left on fur. Numerical parameters useful in detecting the occurrence of these actions are described. Recognition results are verified on the rats' social behaviour thermal images dataset.
\end{abstract}

\section{Introduction}

Automatic recognition of laboratory animals' behaviour has been a very active field of research over the past years. Mouse and rats are being used in a wide range of pre-clinical testing of drugs and diseases. Their social interactions are often a reflection of abnormalities or health condition. However, as far as simple actions, such as speed or direction of movement are quite easy to detect by computer with video camera, more complex behaviours often are challenging.

The first problem of automatic animal behaviour analysis to be tackled is the object detection. Many existing approaches are based on the videos from the top [1, 2] or side [3] of the cage with two or more rodents inside. Proper detection of objects is facilitated by controlled conditions of laboratory cage that often has floor painted in contrast to the animal. One of methods of distinguishing between similar objects is colouring or marking fur. It is also widely used in manual behaviour scoring by human behaviourists. However, situation of body contact and overlapping are still the bottleneck of the detection. And as far as social interactions are concerned, body contact is an essential moment of behaviour analysis.

Another issue to be solved is the selection and detection of significant features of the objects. One of solution is to detect certain parts of the body (snout, base of the tail, centre of gravity), contour or calculate various parameters of the body shape. More recently, recognition tasks are based solely on spatio-temporal features. Presentation of the image as selected points parameters proved to be more effective than the parameters of the whole picture.

Hence, rodents are nocturnal animals with the day (rest) - night (activity) cycle, it is not recommended to disrupt this cycle with artificial lighting, which, on the other hand is necessary for proper recording. Night time is the time of rodents activity and only then some activities can be observed. Dark conditions are also less stressful. For this reason thermal camera is the most optimal choice.

Apart from greater flexibility of the recording conditions, a main advantage of the thermal imaging is information about surface heat distribution of the observed objects. Abnormal body temperature can indicate disease or functional abnormalities of the animal. In [4], [5] and [6] authors proved that fear causes the decrease in skin temperature $\left(-5.3^{\circ} \mathrm{C}\right.$ for the tail [4]), probably due to the peripheral vasoconstriction. Distribution of warmth can also be an individual identifier. Incident of biting one individual by another is difficult to capture even for the human observer. It lasts for a short and occurs during body contact or even occlusion. However, saliva that is rapidly losing temperature remains on the attacked object's fur. It is clearly visible in the thermal images.

Large number of laboratory cages is made from glass or plexiglass, what causes the occurrence of animals reflections in the walls. Thermal imaging is not free of this artefact; however it is easy to cope with due to the much lower temperature of reflections.

Giancardo et al. [2] have monitor two or three mice in a single cage using camera FLIR A315. No artificial tagging of animal were used. They have created a database for a total of $3 \mathrm{~h} 30 \mathrm{~min}$ video recording as well as a system for tracking and behaviour analysis. The tracking algorithm is composed of three modules: detection, detection during body contact and mice matching. Thermal information is used in the last module in case of poor results from the earlier segmentation. For a small number $k$ of previous frames (in experiments $k$ corresponds to 0.3 seconds of video) the temperature distribution inside animal's shapes is observed. That heat signature is then compared to the temperature distribution of each mouse in the current frame with the Kolmogorov-Smirnov test followed by a mouse assignment correction. No other use of the thermal information was presented in the paper.

In this work usage of thermal imaging for rodents social behaviour analysis improvement is presented. The methods to distinguish between individuals and identify particular event on the basis of thermal distribution are tested. 


\subsection{1/qirt.2016.083}

\section{Database}

A rat social interaction database was used. Each time two male rats put inside a cage made of plexiglas for about 20 minutes (figure 1) were recorded. Recordings were made using FLIR A320G camera situated above the cage. The research was performed on two pairs of rats. The first pair differed in size - one of the individual was slightly bigger than the other (body area about $120 \%$ of the smaller individual's area) and in tail temperature (fig 1a). Higher tail temperature can also indicate greater self-confidence (aggressiveness) or lack of fear [4]. Second pair of rodents was more comparable in the size and temperature (fig $1 \mathrm{~b})$.

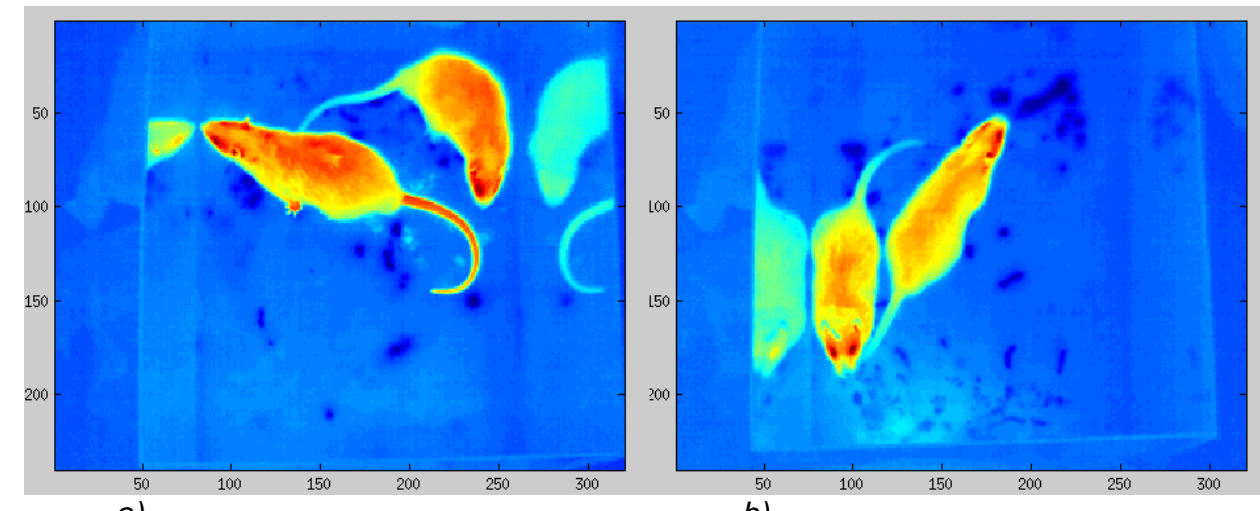

Fig. 1. Frames from database of rats social behaviour test for a) the first and b) second pair of rodents

\section{Temperature distribution object differentiation}

Data analysis based on histogram features is one of the most popular method for image analysis [7].

Object tracking in rodents behaviour analysis is made difficult by moments of connection of objects often with partial or complete covering. It is essential to properly differentiate and track objects after occlusions. To cope with this problem, many researchers use fur markers in the form of colour paint, or patterns made by fur blending or shaving. However, there are no documented researches about lack of influence of above mentioned tagging on animal behaviour. In the visible frequency images two specimens with the same colour are practically undistinguishable. The image of rodents as the distribution of body temperature can be a non-invasive and natural object differentiation parameter [2].

Solution presented in [2] used mice temperature distribution comparison in case of poor results of segmentation. Experiment outcomes introduced below indicate, that rats' thermal distribution can also be a signature of the specimen.

Distribution of body temperature for each rat from social behaviour database failed in test for normal distribution. To compare both distributions non-parametric tests, Mann-Whitney U-test and two-sample Kolmogorov-Smirnov, were used.

Figure 2 presents the results of temperature distribution analysis for each object. Figure $2 a$ is an image of temperature distribution of the whole research scene, figure $2 \mathrm{~b}$ describes and distinguishes between detected objects, figure $2 c$ shows the surface temperature of analysed body area only, figures $2 \mathrm{~d}$ and $2 \mathrm{e}$ are the histograms of temperature in each interesting area.

The $p$-values of Mann-Whitney U-test and two sample Kolmogorov-Smirnov tests for frame in figure 2 are $p<2 \cdot 10^{-10}$ and $p<2 \cdot 10^{-15}$ respectively. Both indicate that the null hypothesis that the data are from the same distribution, can be rejected at the $5 \%$ significance level. 


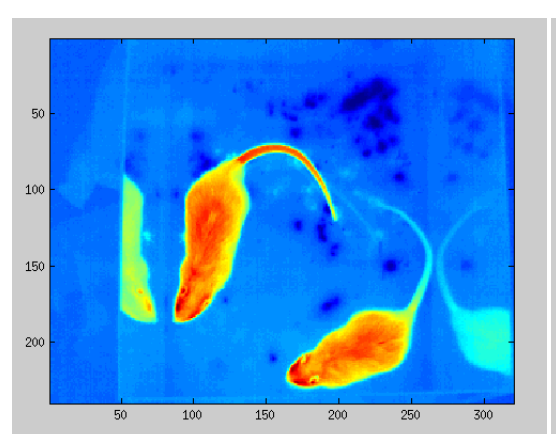

a)

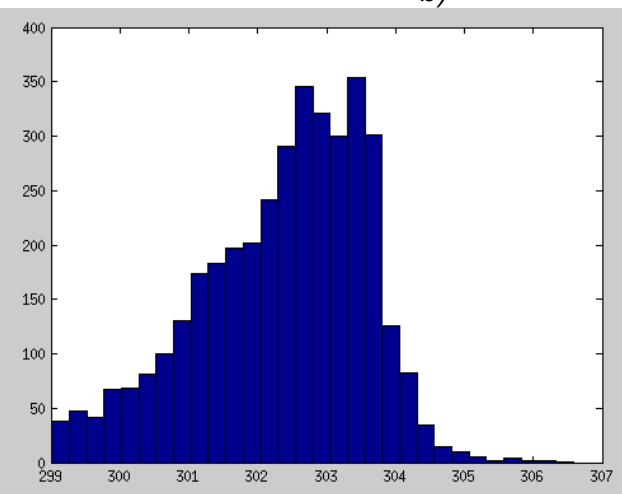

d)

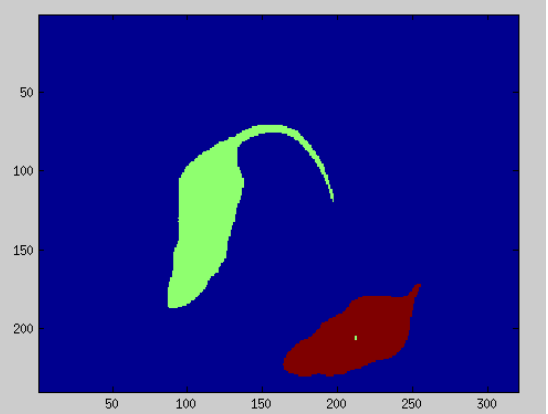

b)

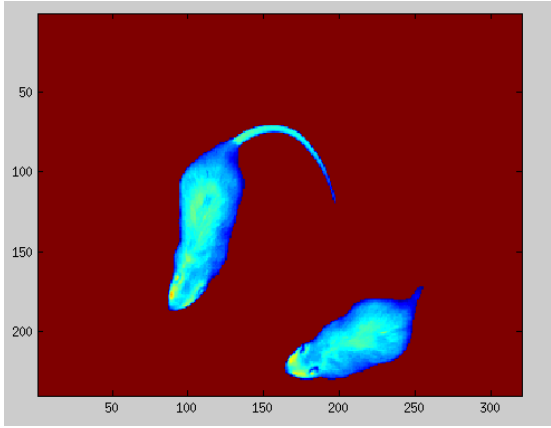

c)

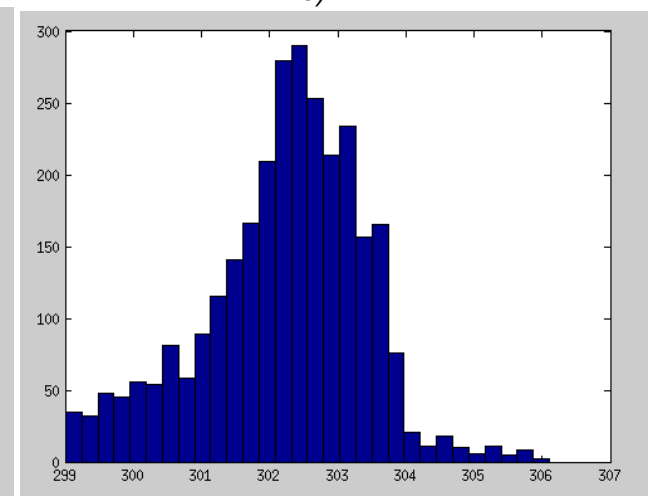

e)

Fig. 2. Results of temperature distribution analysis: a) temperature distribution of the whole research scene, b) detected objects, c) surface temperature of interesting area, d) histogram of temperature [K] of the green specimen, e) histogram of temperature $[K]$ of the red specimen.

Table 1 shows exemplary results of the non-parametric statistical tests with the $5 \%$ significance level for each pair of detected objects. Value 1 in the third column indicates that Mann-Witney U-test rejects the null hypothesis about equal medians of the data distributions. Value 1 in the fifth column indicates that results of two-sample KolmogorovSmirnov test reject the null hypothesis and accept the alternative hypothesis that data are from different distributions.

Table 1. Exemplary results of the two individuals temperature distributions non-parametric tests

\begin{tabular}{|c|c|c|c|c|}
\hline $\begin{array}{c}\text { No. of } \\
\text { frame }\end{array}$ & $\begin{array}{c}\text { p-value for } \\
\text { Mann-Whitney } \\
\text { U-test }\end{array}$ & $\begin{array}{c}\text { Hypothesis of } \\
\text { Mann-Whitney } \\
\text { U-test }\end{array}$ & $\begin{array}{c}\text { p-value for } \\
\text { two-sample } \\
\text { Kolmogorov- } \\
\text { Smirnov test }\end{array}$ & $\begin{array}{c}\text { Hypothesis of } \\
\text { two-sample } \\
\text { Kolmogorov- } \\
\text { Smirnov test }\end{array}$ \\
\hline 20 & $7.093 \cdot 10^{-6}$ & 1 & $1.058 \cdot 10^{-20}$ & 1 \\
\hline 25 & 0.287 & 0 & $2.218 \cdot 10^{-10}$ & 1 \\
\hline 120 & $2.471 \cdot 10^{-18}$ & 1 & $6.366 \cdot 10^{-90}$ & 1 \\
\hline 70 & $3.041 \cdot 10^{-1}$ & 1 & $3.538 \cdot 10^{-19}$ & 1 \\
\hline 112 & $6.063 \cdot 10^{-63}$ & 1 & $1.800 \cdot 10^{-54}$ & 1 \\
\hline 118 & 0.158 & 0 & $2.562 \cdot 10^{-5}$ & 1 \\
\hline
\end{tabular}

Total number of null hypothesis rejection for Mann-Whitney U-test was $98.2 \%$ of all cases for the first pair and $91.4 \%$ for the second. Two-sample Kolmogorov-Smirnov test accepted alternative hypothesis for $100 \%$ and $99.8 \%$ of all cases respectively. Relatively small results for the second pair of Mann-Whitney U-test was probably due to the small differences in size or tail temperature between second individuals. Two sample Kolmogorov-Smirnov test is less sensitive for this kind of variations, however it's $p$-value for cases, where Mann-Whitney U-test failed to reject the null hypothesis, was increasing.

\section{Event identification}

Events that best defines the level of dominance of each individual are aggressive behaviours. Among them biting is one of the most essential in scoring the aggression level, however hard to notice in standard recordings. The goal of attacking rodent is to deliver a bite usually to the subordinate individual's ramp, and the goal of the defender is to prevent this attack. Other aggressive behaviour is offensive neck grooming, when attacker grabs with his teeth other 


\subsection{1/qirt.2016.083}

individual's skin folds and gives rapid little nibbles [8]. All aggressive events appear in not only subjects' close contact but often also while mounting a defender by aggressor, what causes limitation in the field of event observation for a camcorder as well as for a human observer.

Figure 3 shows thermal images taken after (self) grooming (figure 3a) and biting (figure 3b) by another individual. Cool (blue) dots on the fur are the traces of saliva and remain for about 0.5 second until become indistinguishable from the rest of the body. It is not know how long it takes to cool the saliva to the discernible level after biting, as the precise time of biting is almost impossible to observe.

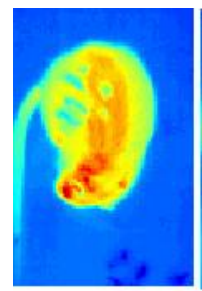

a)

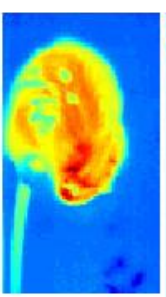

b)

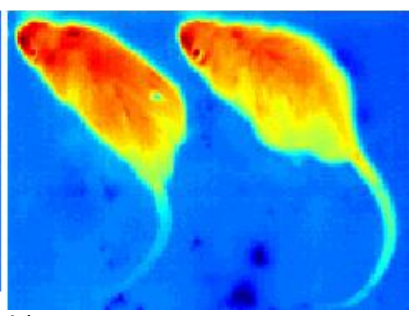

Fig. 3. Traces of saliva with lower temperature than rat's body after a) grooming and b) biting

Recently used image analysis method of representing an image as a set of interesting points can be an interesting solution that brings new information to the rodents' social behaviour analysis when applied to the thermal images. The decision about similarity of the two behaviours is replaced then with corresponding detection and description of a set of features, followed by classification.

In this paper the most popular interesting points detectors have been tested in order to determine their suitability for the analysis of aggressive rodent behaviour.

\subsection{Interesting point detectors}

Three basic detectors of corners were chosen for the test: Harris [9], Shi-Tomasi [10] and FAST [11] algorithms.

Harris algorithm determines a covariance matrix of the intensity gradient for each pixel and its eigenvalues $\lambda_{1}$ and $\lambda_{2}$. An interest point is recognized as a corner if the $R$ value characterised by Eq. (1) is greater than fixed threshold.

where $\mathrm{k}$ is a tunable sensitivity parameter.

$$
\mathrm{R}=\lambda_{1} \lambda_{2}-\mathrm{k}\left(\lambda_{1}+\lambda_{2}\right)^{2}
$$

Shi and Tomasi have modified Harris algorithm by changing the way of decision about corner detection on the basis of intensity gradient covariance matrix eigenvalues to Eq. (2).

$$
\mathrm{R}=\min \left(\lambda_{1} \lambda_{2}\right)
$$

FAST algorithm checks the intensity value of sixteen pixels surrounding the proposed $p$ corner of an intensity of $l$. If among these 16 pixels, there exist $n$ number of pixels that are brighter than $l+$ fixed threshold $t$ or darker than $l-t$, the point $p$ is considered as a corner.

The parameters of algorithms for testing were experimentally selected and equal:

1. Harris:

- $\quad$ minimal accepted quality of corners $(R)=0.0001,0.0005$ and 0.001 ,

$\circ$ the size of Gauss filter $=3,5,7$ and 9 pixels,

2. Shi-Tomasi:

- minimal accepted quality of corners $(R)=0.005,0.01$ and 0.03 ,

$\circ$ the size of Gauss filter $=3,5,7$ and 9 pixels,

3. FAST:

- minimal accepted quality of corners $(R)=0.05,0.1$ and 0.2 ,

$\circ \quad$ minimal difference between intensity of point and his surrounding $=0.01,0.05,0.075$ and 0.1 .

A common feature of selected algorithms is rank calculation of all corners. The lower the rank is, the easier is the point to detect. Figure 4 shows a mean value of detected saliva trace points ranking after bite with different algorithms parameters for three different frames (not following) and three algorithms. Rankings for Harris algorithm are the largest or one of the largest values. It means that respectively 26, 14 and 9 other point are considered as more distinctive. For the first frame the difference between the highest value (Harris algorithm) and the lowest (FAST algorithm) is 14 points. For the third frame Shi-Tomasi and FAST algorithms classify point representing biting trace among top five most characteristic points, while the Harris algorithm among the first ten. 


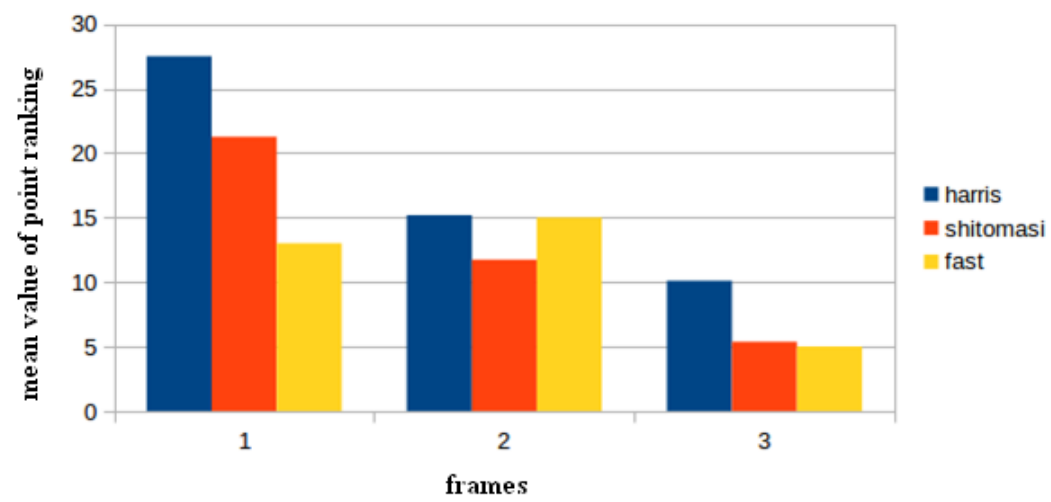

Fig. 4. Mean value of detected saliva trace for three different frames and three detection methods

Table 2 presents the mean values with standard deviation of saliva point ranking from all frames

Table 2. Results of the saliva trace ranking for all frames

\begin{tabular}{|c|c|c|}
\hline Agorithms & Mean value & $\begin{array}{c}\text { Standard } \\
\text { deviation }\end{array}$ \\
\hline Harris & 14.13 & 9.2 \\
\hline Shi-Tomasi & 10.88 & 6.9 \\
\hline FAST & 8.75 & 5.4 \\
\hline
\end{tabular}

Results of total ranking for all algorithms demonstrate that saliva trace is relatively easy to detect and is situated on average in the first 9,11 or 15 interesting points of the image depending on the detector used. The FAST algorithm achieved the best result - rank under 10. The Harris method, in turn, reached rank equal 14 but with highest standard deviation value. Shi-Tomasi method, that is a simplification of Harris ranking, was placed in the middle.

For all three methods interesting corners with higher than saliva trace rank presented primarily other important points like:

- $\quad$ eyes and the inner part of the ear - warmer than skin,

- nose and ear tips - cooler than skin,

- base of the tail.

Rarely they were indicating on the borders of the body.

Figures 5 and 6 presents the results of Harris and FAST algorithms for different frames captured after biting. Figure 5 shows the first a) 5, b) 10 and c) 20 detected points with lowest rank received by Harris algorithm. The saliva trace is marked with a white square in figure 5a). All detected points are marked as black circles. Corners with largest variations in intensity in their neighbourhood according to the Harris ranking appeared to be points of eyes followed by ear tips and nose. The saliva trace was detected as a corner with the rank equal to 17.

Figure 6 presents the first a) 5, b) 10 and c) 15 detected points with lowest rank received from FAST algorithm. The saliva trace is marked with a white square in figure 6a). The first three corners detected by the FAST method turned out to be eyes and ear. These points are clearly visible and have a much higher temperature than the surrounding skin. The saliva trace was in top ten detected points, even though it was not so noticeable in the image. 


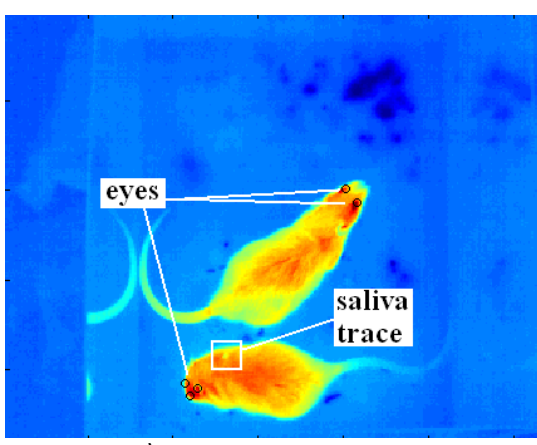

a)

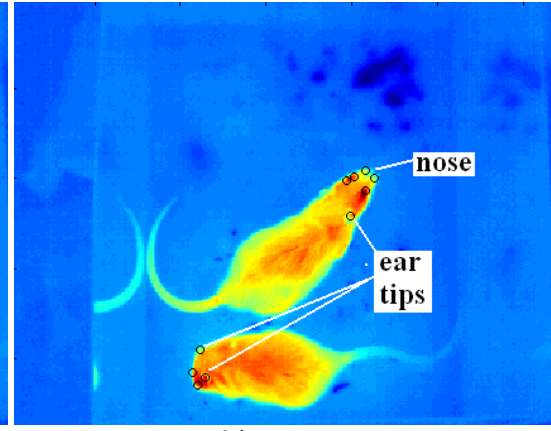

b)

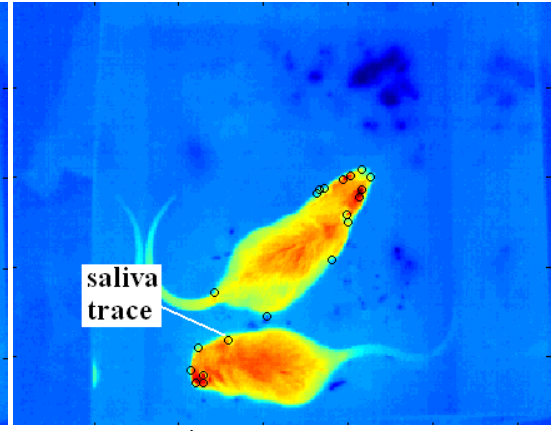

c)

Fig. 5. Results of Harris algorithm for the first a) 5, b) 10 and c) 20 detected corners

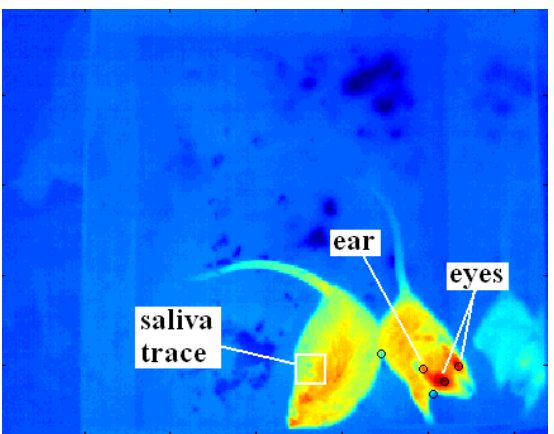

a)

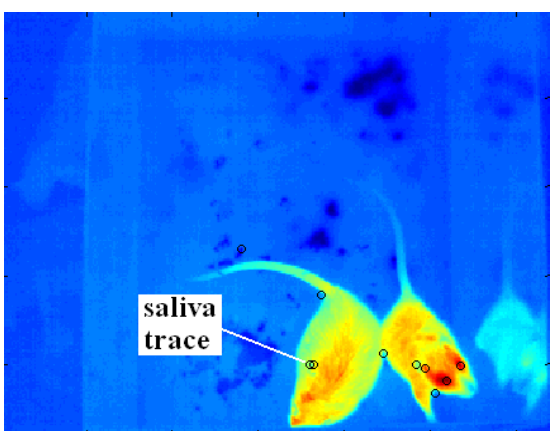

b)

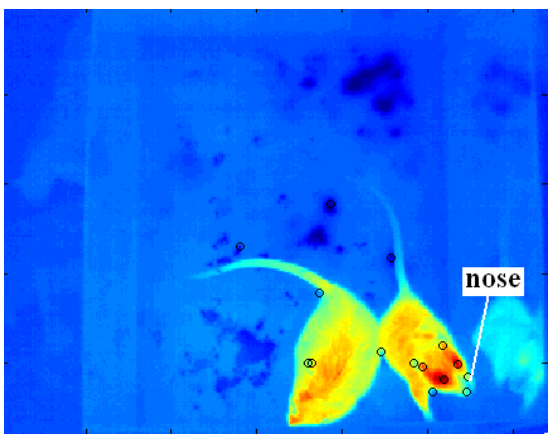

c)

Fig. 6. Results of FAST algorithm for the first a) 5, b) 10 and c) 15 detected corners

\section{Conclusions}

Thermal imaging can introduce important data to rodents social behaviour analysis. Distribution of body surface temperature is proved to be an individual identifier, even for very similar objects. Two-sample Kolmogorov-Smirnov test almost for all examined cases rejected the null hypothesis that distributions of rodents body temperature are from the same distribution. It is possible that slightly different size or temperature value of any body part will significantly simplify discrimination. Emitted thermal radiation can be retained by fur. The variety of the fur density degree probably will also improve individual differentiation.

Popular corners detectors applied for the thermal images of rodents detect windows with the largest variation of pixel intensity, which in fact are eyes, ears, noses and later base of the tails. Traces of saliva left on fur after biting or grooming have surprisingly high results of importance. It is usually detected among first 15 points. Maximal rank in experiments was no higher than 27. Traces of saliva were detected even if are not so clearly visible. The reason of this may be related to the sharp edges of this marks on the fur. Natural temperature distribution of the rodent body is smooth. Edges of extremely high or low temperature, like eyes, nose or ears, are blurred and change gradually. The spot of the saliva on the fur has sharp edges and is not in the contact with warm skin. It remains unchanged until dry. Similar behaviour was noticed only for the tip of the ears in situation when they were visible with the body in the background (figure 6). In this case ear tips have different temperature than neighbourhood and sharp edges and were situated in the top detected corners.

The time of keeping the temperature of saliva much different than the body allows for event observation even after $800 \mathrm{~ms}$. In situation of multiple bites with short intervals between (for example during fight), when detection is complicated by body overlapping and obturation, it is possible to determine the order and timing of bites afterwards on the basis of saliva disappearing moment.

This paper proves that thermal imaging solves some problems of the experimental animal behaviour analysis, particularly in terms of aggressive or social behaviours.

\section{REFERENCES}

[1] Burgos-Artizzu X. P., Dollar P., Lin D., Anderson D. J., Perona P., "Social behavior recognition in continuous video". IEEE Conference on CVPR, pp. 1322-1329, IEEE, Providence, 2012.

[2] Giancardo L., Sona D., Huang H., Sannino S., Manago F., Scheggia D., et. al., "Automatic Visual Tracking and Social Behaviour Analysis with Multiple Mice". PLoS ONE 8(9), September 2013. 


\subsection{1/qirt.2016.083}

[3] Belongie S., Branson K., Dollar P., Rabaud V. "Monitoring animal behavior in the smart vivarium", In Workshop on Measuring Behavior, Wagenigen, The Netherlands. Citeseer, 2005.

[4] Vianna D.M. and Carrive P., Changes in cutaneous and body temperature during and after conditioned fear to context in the rat, Eur J Neurosci. May; 21(9):2505-12, 2005.

[5] Marks A., Vianna D.M.L., Carrive P., Nonshivering thermogenesis without interscapular brown adipose tissue involvement during conditioned fear in the rat, Am J Physiol Regul Integr Comp Physiol 296: R1239 - R1247, 2009.

[6] Thermography (2013). "Current status and advances in livestock animal and veterinary medicine". Edited by $F$. Luzi, M. Mitchell, L.N. Costa and V. Redaelli. Brescia Foundation. Italy. ISBN 978-88-97562-06-1

[7] Sergy S., "Color Histogram Features Based Image Classification in Content-Based Image Retrieval Systems," pp. 221-224, 2008

[8] Miczek, Klaus A. and Seitse F. de Boer. (2004). "Aggressive, defensive, and submissive behavior. In: The behavior of the laboratory rat: a handbook with tests". Ian Q. Whishaw and Bryan Kolb, eds. Oxford University Press, USA.

[9] Harris C. and Stephens M., "A combined corner and edge detector". Proceedings of the 4th Alvey Vision Conference. pp.147-151, 1988.

[10] Shi, J., \& Tomasi, C., "Good features to track". CVPR. Seattle, 1994.

[11] Rosten E. and Drummond T., "Machine learning for high-speed corner detection". European Conference on Computer Vision, May 2006. 